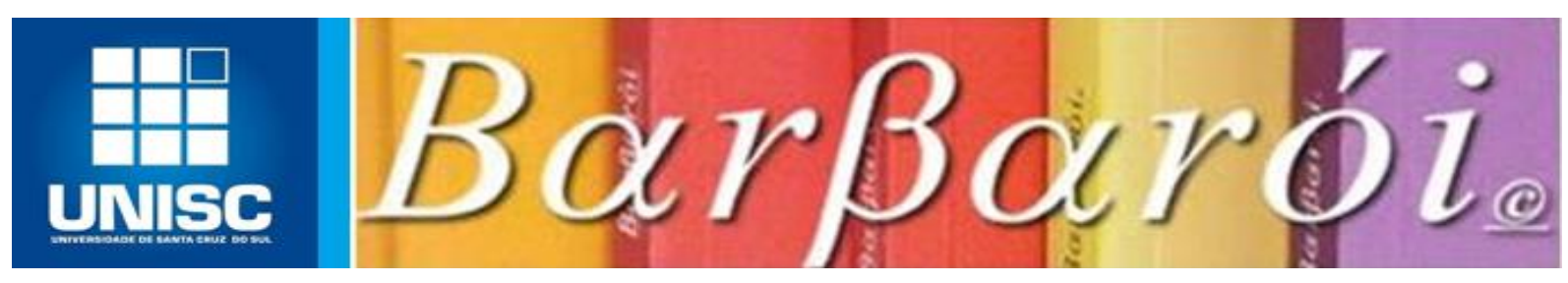

\title{
OFICINAS DE APRESENTAÇÃO ORAL DE TRABALHOS CIENTÍFICOS COM ESTUDANTES UNIVERSITÁRIOS
}

\section{DOI: http://dx.doi.org/10.17058/barbaroi.v0i49.4801}

\author{
$*$ \\ Ana Cristina Garcia Dias \\ Universidade Federal de Santa Maria - UFSM - Brasil \\ Anelise Schaurich dos Santos \\ Universidade Federal de Santa Maria - UFSM - Brasil \\ Clarissa Tochetto de Oliveira \\ Universidade Federal do Rio Grande do Sul - UFRGS - Brasil \\ Maiquel Guilherme Herdina \\ Universidade Federal de Santa Maria - UFSM - Brasil
}

\section{Resumo}

Este estudo descreve a experiência de três oficinas de apresentação oral de trabalhos para estudantes universitários. As oficinas buscavam fornecer informações sobre a preparação e dicas para o dia da apresentação. Participaram das oficinas 33 estudantes de uma universidade pública do sul do Brasil. Os participantes concentravam-se nos semestres iniciais e finais da graduação. Os tópicos abordados envolveram preparação prévia, treino para a apresentação, ansiedade e dia da apresentação. Verificou-se que tanto calouros quanto formandos percebem a necessidade de desenvolver a habilidade de falar em público. Os calouros expressaram o desejo de se preparar para apresentações de trabalhos em sala de aula, enquanto os formandos buscavam maior preparação para apresentação do trabalho de conclusão de curso. Os participantes não pareciam enfrentar problemas no que se referia à preparação do material a ser apresentado e, até mesmo, ao treino da apresentação. A maior preocupação dos estudantes se referia ao manejo da ansiedade em situações de exposição oral. Os participantes temiam esquecer o que falar, falar algo errado e/ou não saber responder as perguntas da plateia ou banca examinadora. As oficinas se constituíram em um espaço para que os estudantes buscassem informações sobre as exposições orais, visto que nem sempre os cursos de graduação e pós-graduação dão conta dessa demanda.

Palavras-chave: estudantes universitários; oficina; apresentação oral.

\section{Introdução}

As apresentações orais estão presentes em diversos momentos da vida acadêmica dos estudantes (LINTE, 2008). Situações como apresentação de trabalhos, avaliações orais e participação durante as aulas requerem que o aluno fale em público (OLIVEIRA; DUARTE, 2004). É comum que discentes participem de congressos, simpósios, seminários, encontros e 
reuniões, os quais possibilitam a divulgação de resultados de estudos e a troca de opiniões sobre determinado assunto. Frequentemente, a exibição dos principais achados de pesquisas e os debates sobre a temática acontecem nas sessões de comunicação oral ou sessões de painéis. As duas modalidades configuram-se como estratégias de divulgação científica realizadas por meio da exposição verbal de tempo variável. Em ambos os casos, o principal recurso utilizado é o próprio corpo do expositor, que inclui os gestos, as expressões faciais, o direcionamento do olhar, a postura e, principalmente, a fala do apresentador (CARMO; PRADO, 2005).

Falar é uma habilidade natural dos humanos. Os indivíduos tendem a aprender em sociedade as diferentes formas de usar a língua nas situações comunicativas concretas (VERDE, 2011). Contudo, seguidamente, as exposições orais acima mencionadas são causa de excessiva ansiedade em diversos estudantes (OLIVEIRA; DUARTE, 2004). Grande parte dos alunos fica apreensiva com a possibilidade de não conseguir se expressar com confiança e fluência nas distintas situações comunicativas com as quais se depara (VERDE, 2011). Essa preocupação pode ser ainda maior quando os acadêmicos percebem que uma apresentação bem sucedida funciona como um "cartão de visitas". O interesse despertado na audiência pode resultar em convites para palestras, cursos ou conferências em eventos científicos, além de ampliar a rede de contatos profissionais (HABIGZANG, 2014).

Em geral, estudantes universitários aprendem a falar em público por tentativa e erro. Raramente, os professores ensinam aos alunos um modelo explícito de retórica (mensagem, público, objetivo e contexto) e oferecem orientações para auxiliá-los a determinar quais conteúdos são relevantes em uma apresentação. Esse cenário pode atrasar o desenvolvimento de habilidades de comunicação efetiva (HABER; LINGARD, 2001). Contudo, a habilidade para apresentar um trabalho oral pode ser desenvolvida (NUNES, RESENDE, PEDROSO, FERREIRA, MACHADO, 2012). A literatura indica que os estudantes sejam instruídos sobre como selecionar o conteúdo com base no contexto da apresentação, sobre os aspectos que contribuem para tornar uma apresentação interessante, a treinar a apresentação antes de realizá-la e a pedir feedback a um instrutor mais experiente (HABER; LINGARD, 2001; KERBY; ROMINE, 2010).

A fim de minimizar as dificuldades encontradas por discentes de uma universidade pública do interior do Rio Grande do Sul na apresentação oral de trabalhos científicos, o projeto de extensão intitulado "Oficinas de Orientação Profissional e Planejamento de Carreira" oferece a "Oficina de Apresentação Oral de Trabalhos" para os alunos da instituição que se interessam em participar da atividade. Tal oficina visa fornecer informações didáticas 
sobre a fase de preparação para uma apresentação e dicas para o dia da apresentação. Essa atividade foi desenvolvida a partir do entendimento de que a comunicação efetiva e as habilidades de apresentação são essenciais para a disseminação dos resultados de pesquisas científicas (WALJEE et al., 2012). Este estudo objetivou relatar a experiência de três oficinas de Apresentação Oral de Trabalhos para universitários, realizadas no período de um ano pelo projeto.

\section{Método}

O projeto de extensão "Oficinas de Orientação Profissional e Planejamento de Carreira" busca desenvolver habilidades importantes para o planejamento da carreira de estudantes universitários. Dentre os assuntos oferecidos pelo projeto, a oficina de Apresentação Oral de Trabalhos foi realizada três vezes no ano de 2013.

No total, as três oficinas contaram com a presença de 33 estudantes de uma universidade pública do Rio Grande do Sul. A idade dos estudantes variou de 18 a 42 anos (média de 23 anos). Os participantes eram alunos dos cursos de Arquivologia (2 estudantes), Desenho Industrial (2), Direito (1), Economia (1), Enfermagem (1), Engenharia Florestal (1), Engenharia Mecânica (1), Filosofia (1), Fisioterapia (1), História (1), Letras (4), Meteorologia (1), Pedagogia (2), Programa Especial de Graduação (1), Produção Editorial (1), Química (3), Relações Internacionais (1), Relações Públicas (1), Serviço Social (2) e Tecnologia em Alimentos (2). Além dos alunos da graduação, as oficinas também contaram com a presença de 2 alunos de pós-graduação em Enfermagem. De modo geral, grande parte dos participantes se encontrava nos semestres iniciais e finais de seus respectivos cursos e a procura foi maior por parte do sexo feminino (78\%).

A divulgação das oficinas foi realizada pelo setor de apoio ao estudante da instituição, através da página virtual da universidade e da página do projeto no Facebook. Os universitários que demonstraram interesse de participar preencheram a ficha de inscrição disponibilizada na internet. As oficinas foram realizadas com os primeiros 20 inscritos a fim de assegurar a efetividade das atividades desenvolvidas.

As oficinas foram realizadas através de uma apresentação de PowerPoint. Os tópicos abordados na oficina e seus objetivos são apresentados na Tabela 1. 
Tabela 1. Objetivos dos tópicos abordados na oficina de apresentação oral de trabalhos

\begin{tabular}{|l|l|}
\hline Tópico/Atividade & Objetivo \\
\hline Preparação prévia & $\begin{array}{l}\text { Explicar aos participantes a necessidade de conhecer o } \\
\text { público alvo de uma apresentação, sua finalidade, os } \\
\text { recursos disponíveis e as formalidades exigidas, assim } \\
\text { como alertar a respeito do design de uma boa } \\
\text { apresentação e sua estrutura propriamente dita. }\end{array}$ \\
\hline Treino para a apresentação & $\begin{array}{l}\text { Explicar aos participantes a necessidade de treinar a } \\
\text { apresentação, para que possam se sentir melhor } \\
\text { preparados e cientes de mudanças que possam ser } \\
\text { necessárias na apresentação para proporcionar melhor } \\
\text { resultado. }\end{array}$ \\
\hline Atividade & $\begin{array}{l}\text { Trabalhar a capacidade dos participantes de falar em } \\
\text { público, dando-lhes alguns minutos para falar sobre um } \\
\text { assunto que dominam. }\end{array}$ \\
\hline Ansiedade & $\begin{array}{l}\text { Explicar aos participantes o que é ansiedade e } \\
\text { proporcionar estratégias comportamentais e cognitivas } \\
\text { para lidar com a mesma. }\end{array}$ \\
prabrentação & $\begin{array}{l}\text { Trabalhar a ideia de chegar com antecedência ao local da } \\
\text { apresentação. São dadas dicas a respeito da apresentação }\end{array}$ \\
\hline
\end{tabular}

As oficinas foram realizadas nas dependências da instituição, entre 17h30min e 19h00min aproximadamente, por ser um horário no qual tanto os alunos de cursos diurnos quanto os alunos de cursos noturnos tendem a não estar envolvidos em atividades letivas. Os participantes também responderam a uma ficha de avaliação com o objetivo de promover melhorias para os próximos encontros.

\section{Resultados e discussão}

A apresentação oral é um meio de comunicação bastante utilizado para a disseminação de informações e do conhecimento no meio científico (FRIED, 2012). Entretanto, são necessárias habilidades de comunicação e de apresentação dos pesquisadores para que a 
informação seja transmitida de forma adequada (WALJEE et al., 2012). A estrutura curricular dos cursos nem sempre oferece conhecimentos teóricos e técnicos além daqueles específicos da profissão (BORGES; MELO, 2007), o que pode resultar em um déficit de habilidades para realização de atividades gerais, como falar em público. Optou-se por oferecer oficinas de apresentação oral de trabalhos científicos com o intuito de criar um espaço para os estudantes universitários terem acesso a informações sobre a fase de preparação e para o dia da apresentação.

Ao contrário do esperado, os participantes das oficinas não se resumiram a estudantes de iniciação científica buscando informações para sua primeira apresentação. O período do curso em que os estudantes se encontraram no momento da realização da oficina indica que tanto calouros quanto formandos percebem a necessidade de desenvolver a habilidade de falar em público. Entretanto, houve diferenças nas expectativas de estudantes de início e final de curso. Os calouros expressaram o desejo de se preparar para apresentações de trabalho em sala de aula e manifestaram dúvidas sobre a divisão do trabalho entre o próprio grupo de alunos e sobre o quanto deveriam falar. Já os formandos buscavam maior preparação para apresentação do trabalho de conclusão de curso e temiam falar algo errado durante a exposição. Essas informações confirmam o quanto a habilidade de falar em público é necessária em diversos contextos da vida acadêmica.

O primeiro tópico abordado na oficina foi a preparação do material que será apresentado. Uma boa apresentação requer a organização de ideias, slides e recursos visuais com antecedência (FRIED, 2012; LINTE, 2008). Para tanto, é necessário verificar se o evento no qual o trabalho será apresentado oferece normas para apresentação (tamanho do pôster, tempo para apresentação), ter em mente quem é a audiência para utilizar linguagem adequada e realizar um esboço da própria apresentação (RODRIGUEZ, 2014). A seleção das informações mais importantes da pesquisa realizada, o resumo das mesmas e a tentativa de torná-las atrativas são essenciais para a elaboração de um pôster, visto que atratividade e legibilidade são as principais características de um pôster de qualidade (FRIED, 2012; RODRIGUEZ, 2014). Então, os participantes das oficinas foram orientados a organizar a apresentação de acordo com os tópicos de um manuscrito (título, autores, afiliação institucional, introdução, método, resultados, discussão, conclusão, referências, agradecimentos e informações para contato) e a selecionar as informações mais importantes de cada tópico. 
Também foi indicado aos estudantes limitar a quantidade de conteúdo por slide. Isso significa dar preferência a imagens que se refiram ao conteúdo que deve ser transmitido e evitar colocar toda a informação em forma de texto (FRIED, 2012; LINTE, 2008). A literatura sobre preparação de apresentações científicas sugere utilizar, no máximo, três itens com ideias por slide no lugar de frases extensas quando o texto não puder ser evitado (FRIED, 2012), já que a maioria das pessoas tende a se lembrar de, aproximadamente, cinco ideias chave (LINTE, 2008). Se o texto for imprescindível, este deve ser escrito em frases curtas, claras e diretas para facilitar a leitura. Mesmo assim, os slides devem funcionar como pistas para a memória dos apresentadores, de forma que possam indicar os conteúdos e exemplos a serem mencionados. Os slides possuem o papel de auxiliar os apresentadores para não perderem a linha de raciocínio, e não competir com o conteúdo apresentado pelos mesmos (HABIGZANG, 2014). Quando se trata de pôster, a indicação é organizar o conteúdo limitando-se a, no máximo, 800 palavras, distribuídas em diferentes blocos com 7 a 11 linhas (RODRIGUEZ, 2014).

O design da apresentação, que envolve cor, fonte e tamanho da letra, deve ser padronizado do início ao fim do material (FRIED, 2012; GREENHALGH; JONGSTE; BRAND, 2011; LINTE, 2008). A escolha de letras escuras sobre um fundo claro tende a facilitar a leitura (HABIGZANG, 2014; RODRIGUEZ, 2014). O apresentador também deve estar atento para a combinação de cores, visto que o objetivo da apresentação é o tema apresentado. A utilização de até três tons da mesma cor evitam contrastes chocantes. A fonte empregada deve contribuir para a legibilidade do conteúdo. As fontes do tipo sans serif (Arial, Helvetica, Gil Sans, Verdana) são as mais indicadas (HABIGZANG, 2014; RODRIGUEZ, 2014). No que se refere ao tamanho da letra para apresentações eletrônicas, o tamanho pode ser 44 pontos para títulos e 24 para o corpo do texto (HABIGZANG, 2014). Para pôster, é recomendado utilizar 85 pontos para o título, 42 para autores, 47 para subtítulos, 34 para texto e 28 para adendos (RODRIGUEZ, 2014).

Para finalizar a discussão sobre a quantidade de conteúdo e o design da apresentação, foi elaborado um slide com texto extenso, escrito em fonte de tamanho pequeno e ilegível, e em cores que dificultavam a visão. Na sequência, solicitou-se aos participantes que relatassem o que havia de errado com aquele slide. Os estudantes apontaram todos os itens trabalhados até então e puderam perceber, na prática, a importância do design da apresentação.

Outro aspecto abordado na oficina foi o cuidado que deve ser dedicado ao tempo de duração da apresentação. Afinal, ultrapassar o limite de tempo estabelecido pelo evento 
reflete preparação deficitária do autor para a apresentação (WAX; CARTIN; PINETTE, 2011). Foi ensinado aos participantes como calcular o tamanho da apresentação de acordo com o tempo disponível. Diversos autores concordam com o cálculo de, pelo menos, um minuto por slide, de forma que uma apresentação de dez minutos deve ter, no máximo, dez slides (GREENHALGH; JONGSTE; BRAND, 2011; HABIGZANG, 2014; WAX; CARTIN; PINETTE, 2011).

Na sequência, foi discutida a importância do treino para uma boa apresentação. Há relatos de que o ensaio pode ser benéfico para o desenvolvimento da habilidade de comunicação, maior controle do tempo, revisão do conteúdo e identificação de lacunas (LINTE, 2008; HABIGZANG, 2014; SOUZA; FERNANDES; FERREIRA, 2013; WAX; CARTIN; PINETTE, 2011). Destacou-se que não há um tempo máximo ou mínimo de treino para uma exposição. O tempo de exercício é o tempo que se tem disponível para isso e depende de cada apresentador (POLITO, 2005). Foi sugerido que os participantes treinassem primeiro sozinhos para, depois, pedirem feedback aos colegas (LINTE, 2008; POLITO, 2005; WAX; CARTIN; PINETTE, 2011). Nesse momento, os estudantes foram convidados a participar de uma atividade com o objetivo de treinar a habilidade de comunicação, já que uma forma eficiente de treinar a expressão oral dos alunos é por meio da exposição oral (VERDE, 2011). Foi explicado aos jovens que as pessoas, geralmente, dominam alguns assuntos que lhe despertam o interesse, como culinária, esportes etc. Então, foi proposto que os participantes que se sentissem à vontade pudessem falar sobre qualquer assunto que dominassem, durante um ou dois minutos, organizado em início, meio e fim. Essa atividade nem sempre foi bem aceita pelos estudantes, possivelmente, devido à timidez por falar em pé, a frente da sala. Em outros encontros, a atividade teve melhor aceitação, apesar de certa resistência inicial. De maneira geral, os participantes consideraram interessante participar dessa atividade, que auxiliou a desmistificar os medos presentes nas apresentações orais e a perceber a possibilidade de serem bem sucedidos nas mesmas.

A observação dos participantes e do conteúdo de seus comentários indicou que a preparação prévia não era o principal interesse dos alunos. Os estudantes que estavam nas oficinas não pareciam enfrentar problemas no que se referia à preparação do material a ser apresentado e, até mesmo, ao treino da apresentação. As sugestões escritas nas fichas de avaliação e as verbalizações dos participantes indicaram que a maior dificuldade dos mesmos consistia no manejo dos pensamentos catastróficos e da ansiedade decorrente destes no 
momento da exposição. A partir disso, a oficina foi reestruturada de forma a abordar o que é a ansiedade e como lidar com ela.

Foi explicado aos participantes que a ansiedade é caracterizada por uma série de reações corporais (palpitação, sudorese, rubor, tontura, náusea etc.) decorrentes da ativação do sistema nervoso simpático. Em outras palavras, o cérebro decodifica determinado estímulo como perigoso e prepara o corpo para lutar ou fugir (LEAHY, 2011). Em situações de apresentação oral, os sintomas de ansiedade são considerados normais e esperados (HABIGZANG, 2014), visto que os apresentadores são, inevitavelmente, avaliados pela audiência. Os estudantes foram questionados sobre a fonte de sua ansiedade, isto é, o que eles têm medo que aconteça. A maioria dos jovens respondeu possuir medo de esquecer o que falar no momento da apresentação, de falar algo errado e/ou de não saber responder as perguntas da plateia ou banca.

Em relação ao medo de esquecer o que falar, foi sugerido aos participantes que, quando isso acontecesse, eles poderiam repetir a última frase que haviam pronunciado, como se quisessem dar ênfase àquela parte da mensagem. Seguidamente, ao reproduzir a frase e chegar ao ponto de esquecimento, a informação perdida surge naturalmente. Outra opção é pronunciar a expressão: "na verdade, o que eu quero dizer é...”. Essa frase obriga o expositor a explicar a mensagem sob outro ângulo, de forma que o pensamento se reorganiza para seguir a sequência planejada. Caso essas estratégias não funcionem, o mais adequado é dizer para a plateia que o aspecto que estava sendo abordado será retomado em seguida e passar para outro tópico da apresentação (POLITO, 2005).

Após, indagou-se qual o problema de que o que os participantes têm medo venha a acontecer, a fim de mostrar que o constrangimento que temem, se de fato se tornar real, é passageiro, e que, em breve, as pessoas irão se esquecer do que ocorreu. Na sequência, foram ensinadas duas técnicas comportamentais para diminuir as sensações corporais causadas pela ansiedade: respiração diafragmática e relaxamento muscular progressivo (LEAHY, 2011). Dessa forma, é possível amenizar o estresse gerado e, assim, diminuir a preocupação ao se realizar uma apresentação.

A respiração diafragmática visa estimular o sistema nervoso autônomo parassimpático para propiciar uma sensação de relaxamento (KAPEZINSKI; MARGIS, 2004). Tal técnica restabelece a adequação de oxigênio e gás carbônico, estimulando o controle sobre o funcionamento cardiovascular acarretando em uma mudança na frequência cardíaca. A pessoa deve inspirar, estendendo o abdome, e expirar contraindo-o. A imaginação de uma vela em 
frente aos lábios que não deve ser apagada ao expirar pode auxiliar a manter o ritmo da respiração (ARAÚJO; DONIDA, 2008). É importante praticar a respiração diafragmática de modo que seja possível, com o tempo, diferenciá-la da respiração torácica, acompanhando os movimentos que envolvem os dois tipos de respiração.

O relaxamento muscular progressivo (RMP) consiste em provocar contrações e relaxamentos sucessivos de grupos musculares, identificando as diferenças entre esses dois estímulos de modo que seja possível perceber precocemente situações de tensão muscular (KAPEZINSKI; MARGIS, 2004). Orienta-se que se contraia a musculatura por 5 segundos e que a relaxe por 10 segundos, alternadamente, de modo que se obtenha um estado de conforto e bem estar (ARAÚJO; DONIDA, 2008). Em um primeiro momento, deve-se aprender a tencionar e a relaxar separadamente os grupos musculares e, depois de provocada a contração, fazer um relaxamento súbito da musculatura. Após adquirir prática na técnica, se reduz gradativamente os grupos musculares utilizados no exercício para oito e, mais adiante, quatro grupos que focalizem as áreas problemáticas durante situações de ansiedade (KAPEZINSKI; MARGIS, 2004).

Assumir o próprio "estado ansiogênico" para o público também pode contribuir para maior controle da ansiedade através da descontração e relaxamento (HABIGZANG, 2014). Além disso, segurar um objeto pode deixar os apresentadores mais seguros, pois elimina a preocupação do que fazer com as mãos. É preferível optar por objetos relacionados ao contexto da apresentação para não desviar a atenção dos ouvintes. Por exemplo, se estiver realizando uma apresentação com recursos audiovisuais, segurar o laser pointer; se estiver realizando uma apresentação com auxílio de quadro branco, segurar um pincel anatômico (POLITO, 2005). Por fim, foi discutido que a exposição dos participantes a situações de apresentação oral os permite testar as previsões catastróficas e aumentar a confiança nas próprias habilidades com a prática.

Infelizmente, não se pode verificar se a melhor compreensão da ansiedade e se a aplicação de técnicas comportamentais, de fato, auxiliaram os participantes da oficina em apresentações orais de trabalho. No entanto, há evidências de que o automonitoramento, a reestruturação cognitiva e o treino em habilidades sociais com estudantes universitários auxiliam na redução dos níveis de ansiedade através da identificação de estímulos aversivos, da percepção dos eventos de forma mais realista e da criação de um repertório mais rico (OLIVEIRA; DUARTE, 2004). 
Na parte final da oficina, foram discutidos aspectos que devem ser atentados no dia e no momento da apresentação. A aparência pessoal não deve ser ignorada, uma vez que o descuido nesse sentido (como roupas informais em ambiente formal) pode prejudicar a credibilidade do apresentador (HABIGZANG, 2014; POLITO, 2005). É importante que o apresentador esteja no local do evento com antecedência para testar os equipamentos audiovisuais que irá utilizar. Foi discutida a importância de ter um "plano B", caso ocorra algum imprevisto. Nesse caso, isso significa ter a apresentação em dispositivos diferentes (pen drive, CD e online) e uma cópia impressa (HABIGZANG, 2014). Os participantes foram orientados a identificarem a si mesmos e os coautores, junto com a titulação e filiação institucional, e a explicar brevemente sobre o que o trabalho trata a fim de atrair a atenção dos ouvintes. A apresentação deve ser clara, objetiva e articulada, de forma que introdução, método, resultados e conclusão sejam coerentes com os objetivos e hipóteses de pesquisa, ressaltando qual a principal contribuição do estudo para o avanço do conhecimento. A conclusão, em especial, pode incluir um resumo para retomar as principais informações apresentadas, sugestões sobre como o conhecimento pode ser aplicado pelo público em sua prática profissional e pesquisas futuras e, ainda, a mensagem final, com destaque para a principal contribuição (teórica, metodológica, prática) do estudo para o avanço da ciência (HABIGZANG, 2014).

Há comportamentos que podem contribuir para a qualidade da apresentação. O estabelecimento e manutenção de contato visual com os ouvintes, o entusiasmo para falar sobre a pesquisa, a linguagem adotada e a expressão corporal podem auxiliar no engajamento do público (HABIGZANG, 2014; LINTE, 2008; POLITO, 2005; WALJEE et al., 2012). O contato visual com a audiência demonstra domínio sobre a apresentação, uma vez que o apresentador não precisa recorrer a anotações. Além disso, permite ao apresentador identificar o nível de interesse e compreensão do público através da observação de comportamentos, como bocejar, adormecer, balançar a cabeça positivamente, sorrir. O entusiasmo refere-se ao interesse pelo tema apresentado e pode ser potencializado pela postura, sorriso, respiração controlada e movimentação não excessiva. A linguagem utilizada deve ser adequada ao público, evitando jargões, gírias e vícios de linguagem (“tá”, “né”, “ahn”) (HABIGZANG, 2014; POLITO, 2005). Também é importante impor ritmo à exposição, uma vez que falar sempre com a mesma velocidade e o mesmo volume, sem inflexão da voz que acentue de maneira apropriada as pausas expressivas das frases pode fazer com que o público perca o interesse pela comunicação (POLITO, 2005). A expressão corporal adequada diz respeito à 
execução de gestos moderados, os quais devem acompanhar de maneira harmoniosa o ritmo da fala. Deve-se ter cuidado para não permanecer todo o tempo da fala com as mãos nos bolsos, com os braços cruzados ou presos nas costas. Ademais, não é adequado se movimentar de um lado para o outro sem objetivos (POLITO, 2005).

No que se refere ao período das perguntas ao final da apresentação, a literatura oferece algumas opções sobre como lidar com questões que o autor não sabe responder, uma das principais preocupações dos participantes das oficinas. Em primeiro lugar, é educado agradecer todas as pessoas que perguntarem algo sobre o trabalho apresentado. Além disso, essa atitude estimula a participação e fortalece o vínculo com o público (HABIGZANG, 2014). Se o autor não souber a resposta, deve ser honesto e admitir que não sabe, perguntando, em seguida, se alguém da plateia pode ajudar a responder a questão (LINTE, 2008; GREENHALGH; JONGSTE; BRAND, 2011). A compreensão de que algumas pessoas perguntam porque realmente estão interessadas no trabalho que está sendo apresentado e que outras o fazem para falar da pesquisa que estão desenvolvendo pode auxiliar os estudantes a lidarem com as questões que podem emergir no momento final da apresentação. As pessoas interessadas tendem a fazer perguntas interessantes e fáceis de responder, enquanto as outras tendem a realizar perguntas longas e irrelevantes. Nesse caso, uma boa resposta pode ser: “Agradeço pela pergunta, mas, infelizmente, esse aspecto não foi investigado porque vai além dos objetivos desta pesquisa" (GREENHALGH; JONGSTE; BRAND, 2011). Mesmo assim, recomenda-se a preparação para possíveis perguntas, como a escolha de determinado método e não outro, com respostas que justifiquem a escolha de forma pontual.

A análise das fichas de avaliação preenchidas pelos participantes permitiu identificar, de forma objetiva, o nível de satisfação dos estudantes no que se refere aos encontros realizados. A maioria dos estudantes (70,4\%) afirmou que a oficina alcançou totalmente suas expectativas e que participaria de outro encontro no futuro $(77,8 \%)$. Esses resultados indicam que as oficinas oferecem um panorama geral dos aspectos importantes a serem considerados no planejamento e na realização de uma apresentação oral de trabalhos científicos. Contudo, a duração da mesma e o desconhecimento do perfil dos estudantes interessados em participar a cada encontro limitam a exploração mais aprofundada de fatores mais relevantes para aqueles com experiência de comunicações científicas. 


\section{Considerações finais}

O objetivo deste estudo foi relatar a experiência de três oficinas de Apresentação Oral de Trabalhos para universitários, realizadas no período de um ano. Os comentários dos partipantes durante as oficinas sugeriram que as exposições orais, tanto em sala de aula quanto em congressos, simpósios, seminários, encontros e reuniões, são momentos ansiogênicos para estudantes de graduação e pós-graduação. Tanto isso é verdade que os participantes demonstraram mais interesse no tópico da oficina que tratava sobre a ansiedade e as possíveis soluções para minimizá-la nos dias das apresentações.

Uma das deficiências da oficina é o fato de ela ser executada em apenas um encontro com duração de aproximadamente uma hora e trinta minutos. O tempo de duração não possibilita o aprofundamento dos conteúdos trabalhos. Ademais, um único encontro não permite constatar a efetividade das oficinas na mudança de comportamento dos participantes. É possível que a realização da atividade de forma processual, composta por mais encontros, seja mais efetiva para o desenvolvimento das habilidades necessárias para apresentações de trabalhos.

Outra carência é a realização da mesma oficina para estudantes de diversos cursos e semestres. Possivelmente, áreas do conhecimento diferentes requerem conhecimentos distintos sobre apresentações orais. Além disso, alunos dos semestres finais e de pósgraduação podem ter expectativas diferentes dos universitários iniciantes em relação aos assuntos abordados nas oficinas. Contudo, a preparação de uma oficina específica em função das características do grupo de participantes não é possível, já que a equipe de extensão é reduzida e não daria conta de tamanha demanda.

Com a realização das oficinas, notou-se que os cursos de graduação e pós-graduação não parecem destinar um espaço na grade curricular para orientar os alunos a respeito das apresentações de trabalhos. Os estudantes devem buscar informações sobre as exposições orais por conta própria e aprender sozinhos o que fazer e o que não fazer no momento das comunicações. Acredita-se que as Oficinas de Apresentação Oral de Trabalhos, promovidas pelo projeto de extensão "Oficinas de Orientação Profissional e Planejamento de Carreira", concedem um espaço para que os discentes debatam sobre o assunto e apreendam dicas para uma comunicação e o dia da apresentação. Assim, a principal contribuição deste estudo é auxiliar na criação de oficinas similares a essas em outras instituições de ensino superior, 
destacando a necessidade de promover o desenvolvimento de habilidades pessoais dos alunos, além dos conhecimentos técnicos.

\title{
WORKSHOPS ON ORAL SCIENTIFIC PRESENTATIONS WITH COLLEGE STUDENTS
}

\begin{abstract}
This study describes the experience of three workshops on oral scientific presentations with college students. The workshops aimed to provide information on how to prepare an oral presentation as well as to give some tips on how to present the work. The participants were 33 students from a public university of southern Brazil. Most participants were in freshman and senior year. The topics included prior preparation, training for scientific presentation, anxiety, and the day of scientific presentation. We noticed that both freshmen and seniors realized it was an important skill to develop the ability to speak in public. Freshmen expressed motivation to be prepared for paper presentations in their classes, while seniors wanted to be prepared for the presentation of their final report. Participants do not seem to face problems related to the preparation of the material to be presented, nor were problems presented for the training of scientific presentation. Students' greatest concern refers to anxiety management due to oral exposure. The students were afraid of forgetting what to say, saying something wrong and/or not knowing how to answer the audience or examiners. The workshops consist of an activity where students can seek for information about oral presentation, since undergraduate and graduate courses do not often meet this demand.
\end{abstract}

Keywords: college students; workshop; oral presentation.

\section{TALLERES DE PRESENTACIÓN ORAL DE TRABAJOS CIENTÍFICOS CON ESTUDIANTES UNIVERSITARIOS}

\section{Resumen}

Este estudio describe la experiencia de tres talleres de presentación oral de trabajos para estudiantes universitarios. Los talleres intentaban proveer informaciones sobre la preparación y consejos para el día de la presentación. Participaron de los talleres 33 estudiantes de una universidad pública del sur de Brasil. La mayor parte de los estudiantes estaban en los primeros y en los últimos semestres de la carrera. Los temas abordados involucraron preparación previa, entrenamiento para la presentación, ansiedad y día de la presentación. Se verificó que tanto novatos como los que están por egresar perciben la necesidad de desarrollar la habilidad de hablar en público. Los novatos expresan el anhelo de prepararse para presentaciones de trabajos en clase, ya los concluyentes buscan más preparación para la presentación de la tesina. Los estudiantes no parecían tener problemas respecto a la preparación del material que presentarían e, incluso, al entrenamiento de la presentación. La mayor preocupación de los estudiantes se refería al manejo de la ansiedad en situaciones de exposición oral. Los participantes temían olvidar que decir, hablar algo mal y/o no saber responder a las preguntas de la platea o comisión examinadora. Los talleres se han vuelto un espacio donde los estudiantes buscan informaciones sobre las exposiciones orales, pues no siempre los cursos de graduación y posgrado cubren esa demanda.

Palabras-clave: estudiantes universitarios; taller; presentación oral. 


\section{Referências}

ARAÚJO, R. B.; DONIDA, D. T. Técnicas em terapia cognitivo-comportamental. In: PICCOLOTO, L.; PICCOLOTO, N. M.; WAINER, R. Tópicos especiais em terapia cognitivo-comportamental. São Paulo: Casa do Psicólogo, 2008.

BORGES, L. O.; MELO, S. L. A transição da universidade ao mercado de trabalho na ótica do jovem. Psicologia: Ciência e Profissão, v. 27, n. 3, p. 376-395, 2007.

CARMO, J. S.; PRADO, P. S. T. Apresentação de trabalho em eventos científicos: comunicação oral e painéis. Interação em Psicologia, v. 9, n. 1, p. 131-142, 2005.

FRIED, Jacquelyn. Techniques for professional presentation of scientific information. The Journal of Dental Hygiene, v. 86, n. 1, p. 24-25, 2012.

GREENHAlGH, T.; JONGSTE, J. C.; BRAND, P. L. P. Preparing and delivering a 10minute presentation at a scientific meeting. Paediatric Respiratory Reviews, v. 12, p. 148149, 2011.

HABER, R. J.; LINGARD, L. A. Learning oral presentation skills. Journal of General Internal Medicine, v. 16, n. 5, p. 308-314, 2001.

HABIGZANG, L. F. Como preparar e realizar apresentações orais. In: KOLLER, S. H.; PAULA COUTO, M. C. P.; HOHENDORFF, J. V. (Org.). Manual de produção científica. Porto Alegre: Penso, 2014.

KAPEZINSKI, F.; MARGIS, R. Transtorno de ansiedade generalizada. In: KNAPP, P. Terapia cognitivo-comportamental na prática psiquiátrica. Porto Alegre: Artmed, 2004.

KERBY, D.; ROMINE, J. Develop oral presentation skills through accounting curriculum design and course-embedded assessment. Journal of Education for Business, v. 85, n. 3, p. 172-179, 2010.

LEAHY, R. L. Livre de ansiedade. Porto Alegre: Artmed, 2011.

LINTE, C. A. The art of dissemination: what makes an effective scientific presentation? IEEE Engineering in Medicine and Biology Magazine, v. 27, n. 4, p. 5-9, 2008.

NUNES, F. D. D.; RESENDE, K. A.; PEDROSO, S.; FERREIRA, T. C.; MACHADO, R. M. "Da loucura a ciência" Oficinas de oralidade e comunicação estratégica. Revista de Enfermagem da UFSM, v. 2, n. 1, p. 577-583, 2012.

OlIVEIRA, M. A.; DUARTE, A. M. M. Controle de respostas de ansiedade em universitários em situações de exposições orais. Revista Brasileira de Terapia Comportamental e Cognitiva, v. 6, n. 2, p. 183-199, 2004.

POLITO, R. Superdicas para falar bem em conversas e apresentações. São Paulo: Saraiva, 2005 . 
RODRIGUEZ, S. N. Como preparar um pôster científico. In: KOLLER, S. H.; PAULA COUTO, M. C. P.; HOHENDORFF, J. V. (Org.). Manual de produção científica. Porto Alegre: Penso, 2014.

SOUZA, R. A. S. A.; FERNANDES, A. C. N.; FERREIRA, L. P. Oficina de expressividade para universitários em situação de apresentação de seminários. Distúrbios da comunicação, v. 25, n.3, p. 458-476, 2013.

VERDE, C. M. F. C. A comunicação oral na sala de aula. 2011. 128 f. Dissertação (Faculdade de Ciências Sociais e Humanas - Mestrado) - Universidade Nova de Lisboa, Lisboa, 2011.

WALJEE, J. F. et al. Developing the art of scientific presentation. The Journal of Hand Surgery, v. 37, n. 12, p. 2580-2588, 2012.

WAX, J. R.; CARTIN, A.; PINETTE, M. G. Preparing a research presentation: a guide for investigators. American Journal of Obstetrics and Gynecology, v. 205, n. 28, p. 1-5, 2011.

Data de recebimento: 01/06/2014

Data de aceite: 01/11/2017

\section{Sobre os autores:}

Ana Cristina Garcia Dias é Psicóloga, Doutora em Psicologia Universidade de São Paulo/SP, Professora dos Programas de Pós-graduação em Psicologia da Universidade Federal do Rio Grande do Sul e da Universidade Federal de Santa Maria. Endereço Eletrônico: anacristinagarciadias@gmail.com

Anelise Schaurich dos Santos é psicóloga (Universidade Federal de Santa Maria UFSM), Especialista em Gestão em Saúde (UFSM), Mestre em Psicologia (UFSM), discente de Doutorado do Programa de Pós-Graduação em Psicologia da Universidade do Vale do Rio dos Sinos (Unisinos) com bolsa CAPES/PROSUP e docente dos cursos de Psicologia e Administração da SOBRESP - Faculdade de Ciências da Saúde. Endereço Eletrônico: anelise_ssantos@hotmail.com

Clarissa Tochetto de Oliveira possui graduação e mestrado em Psicologia pela Universidade Federal de Santa Maria e especialização em psicoterapia cognitivo-comportamental pela Wainer \& Piccoloto. Atualmente, é doutoranda em Psicologia na Universidade Federal do Rio Grande do Sul e bolsista CNPq. Endereço Eletrônico: clarissa.tochetto@ gmail.com

Maiquel Guilherme Herdina é Graduado em Psicologia pela Universidade Federal de Santa Maria. Endereço Eletrônico: maiquelherdina@gmail.com 